\title{
Implementing Automatic Location Update for Follow-Me Database Using VolP and Bluetooth Technologies
}

\author{
Yi-Bing Lin, Senior Member, IEEE, Hsu-Yung Cheng, \\ Ya-Hsing Cheng, and Prathima Agrawal, Fellow, IEEE
}

\begin{abstract}
Personal Number (PN) service or Follow-me service allows a user to access telecommunication services with any terminals (e.g., fixed telephones or mobile phones) in any locations within the service area. To provide this feature, the PN user needs to manually register with a phone number every time he/she enters a new location. If the user forgets to register the new phone number, the incoming calls will be misrouted. To provide user-friendly Follow-me service, this paper proposes an Automatic Follow-me Service (AFS) approach that automatically updates the PN records in the Follow-me database. The significance of our approach is that AFS can be integrated with existing Follow-me databases to automate PN services offered by different PSTN service providers. We show how AFS can be implemented by using the Voice over IP and Bluetooth technologies. Then, we propose an analytic model to investigate the performance of AFS. The analytic results are validated by simulation experiments. Our study suggests how to select polling frequency to optimize the AFS performance.
\end{abstract}

Index Terms-Bluetooth, Follow-me database, location update, personal number, polling, Voice over IP.

\section{INTRODUCTION}

$\mathrm{P}$ ERSONAL NUMBER (PN) service, or "Follow-me" service [10], supports personal mobility, which allows a PN user to access telecommunication services with any terminals (e.g., fixed telephones or mobile phones) in any locations within the service area. This feature is achieved by using a Follow-me database. When moving into a new location, a PN user should register to the Follow-me database so that the public switched telephone network (PSTN) service provider can locate the telephone where the user resides. In this approach, a PN user should register to the Follow-me database so that the public switched telephone network (PSTN) service provider can locate the telephone where the user resides. In this approach, the PN user needs to manually register with a different phone number every time he/she enters a new location. If the user forgets the registration procedure, the incoming calls will be misrouted. The misrouting effect of manual registration has been investigated in [17]. It is clear that the manual registration procedure is very inconvenient. To provide user-friendly Follow-me service, this paper proposes an Automatic Follow-me Service (AFS) approach that automatically updates the PN records in the Follow-me database.

- Y.-B. Li, H.-Y. Cheng, and Y.-H. Cheng are with the Department of Computer Science and Information Engineering, National Chiao Tung University, Hsinchu, Taiwna, ROC.

E-mail: \{liny, chengsy, yscheng\}@csie.nctu.edu.tw.

- P. Agrawal is with Telcordia Technologies, 445 South Street, MCC 1J244B, Morristown, NJ 07960-6438.

E-mail: pagrawal@research.telcordia.com.

Manuscript received 15 July 2001; revised 15 May 2002; accepted 20 May 2002.

For information on obtaining reprints of this article, please send e-mail to: tc@computer.org, and reference IEEECS Log Number 116588.
The AFS architecture is illustrated in Fig. 1. In this architecture, the Follow-me service is provided by the PSTN service provider. The AFS system consists of two parts: a private telephony system and a radio tracking system. The private telephony system can be a private branch exchange (PBX) or a private voice over IP (VoIP) network, which interacts with the Follow-me database to provide location information of a PN user. The radio tracking system utilizes wireless technology such as DECT [5] or Bluetooth [15], which detects the location of a user and reports it to the private telephone system. In our implementation, the private telephony system is a VoIP network. The VoIP network consists of VoIP gateways (Fig. 1(1)) and a call agent (Fig. 1(2)). The gateway is responsible for voice transmission and the call agent is responsible for call control. Details of the VoIP network will be elaborated in Section 2. The radio tracking system is based on Bluetooth technology, which consists of Bluetooth access points (APs; see Fig. 1(3)) connecting to the VoIP gateways. Bluetooth is chosen because it is anticipated that most information appliances will be equipped with Bluetooth capability in the future. A Bluetooth portable device (PD; see Fig. 1(4)) can be carried by a PN user for the AFS tracking purpose. The PD can be a location badge, a personal digital assistant (PDA), or a mobile phone. The AP tracks the location of a mobile user by polling the PD and reports the corresponding telephone number of the location to the Follow-me database (Fig. 1(5)) through the VoIP network. Two AFS scenarios are described as follows:

Scenario I. The user does not carry any mobile phone and the PD is a location badge or a PDA. Consider the example in Fig. 2 where office- 1 and office- 2 are covered by AFS. Suppose that a user is in office- 1 initially and the 


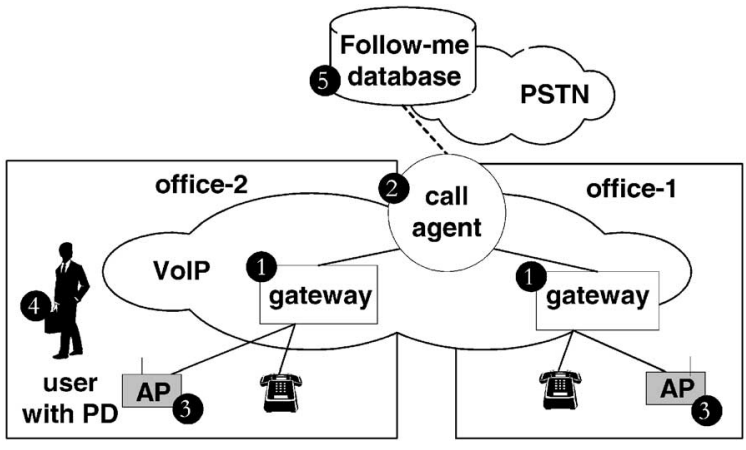

Fig. 1. Automatic Follow-me service architecture.

Follow-me database records the number num-1 of a fixed phone in office-1. When the user moves from office-1 to office-2, the Bluetooth access point AP2 (Fig. 2(1)) detects that the user has entered office-2. AP2 reports the number of a near-by telephone (i.e., num-2) to the AFS. The AFS executes the Registration procedure (to be elaborated in Section 2) to update the Follow-me database through the VoIP network Fig. 2(2)). The user's telephone number in the Follow-me database (Fig. 2(3)) is then modified from num-1 to num-2. If the user leaves office- 2 and enters an area not covered by AFS, AP2 will detect the absence of the user through the polling mechanism (to be elaborated in Section 3). In this case, the telephone number of the user in the Follow-me database is set to a default number, which is, for example, the secretary's phone number (num-3 in Fig. 2). When a person dials the PN of the user, the PSTN delivers the call according to the PN record in the Follow-me database. In Fig. 2, if the user is in office-2, the call will be delivered to phone-2. When the user is out of the AFS service area, the call is delivered to the secretary of the user.

Scenario II. The PD is a portable phone with Bluetooth capability (which is called a three-in-one phone in the Bluetooth usage model [3]). The PD can be used as a cellular phone in the standard manner, as a cordless phone connecting to a Bluetooth $\mathrm{AP}$, and as an intercom or "walkie-talkie" for direct phone-to-phone communications with another three-in-one phone in proximity. In this AFS scenario, three numbers are assigned to the PD:

- The PN number is used by other people to access the PN user.

- The cellular phone number num-CP is used when the PD functions as a cellular phone.

- The Bluetooth number num-BT is used when the $\mathrm{PD}$ acts as a Bluetooth cordless phone.

As shown in Fig. 3, a Location Register (LR) is maintained in the private VoIP network. The LR maps num-BT to the address of the AP where the user resides. If the user is out of the AFS service area, num-BT in the LR is mapped to a void AP number. Consider the example in Fig. 3 . The user is in office- 1 initially. The Follow-me database maps the PN number to num-BT and the LR maps num-BT to access point AP1. When someone dials the PN number, the PSTN obtains num-BT by querying the Follow-me database. The PSTN then attempts to connect to num-BT, which results in a call path setup to the AFS VoIP network. By querying the LR, the AFS identifies that the PD is covered by AP1 and instructs AP1 to page the PD. When the PD responds, the call is connected. In this case, the PD is a Bluetooth cordless phone. When the user moves from office- 1 to office-2, the situation is detected by AP2. AP2 informs the AFS of this movement. The LR then maps num-BT to AP2. No action needs to be taken to update the Follow-me database. When the user moves from office- 2 to an area not covered by AFS, AP2 will detect the situation from the polling mechanism. The AFS maps num-BT to "void" in the LR and sets the PN record in the Follow-me database to num-CP (this is achieved by automatically dialing a PN number modification call to the PSTN; see Section 2). Outside the AFS enterprise area, the telecom are provided by cellular service providers. That is, when someone dials the user's PN number, the PSTN obtains num-PC from the Followme database and sets up the call to the gateway mobile switching center of the cellular network. The call is then delivered following the standard mobile termination procedure [14]. In this case, the three-in-one phone functions as a cellular phone.

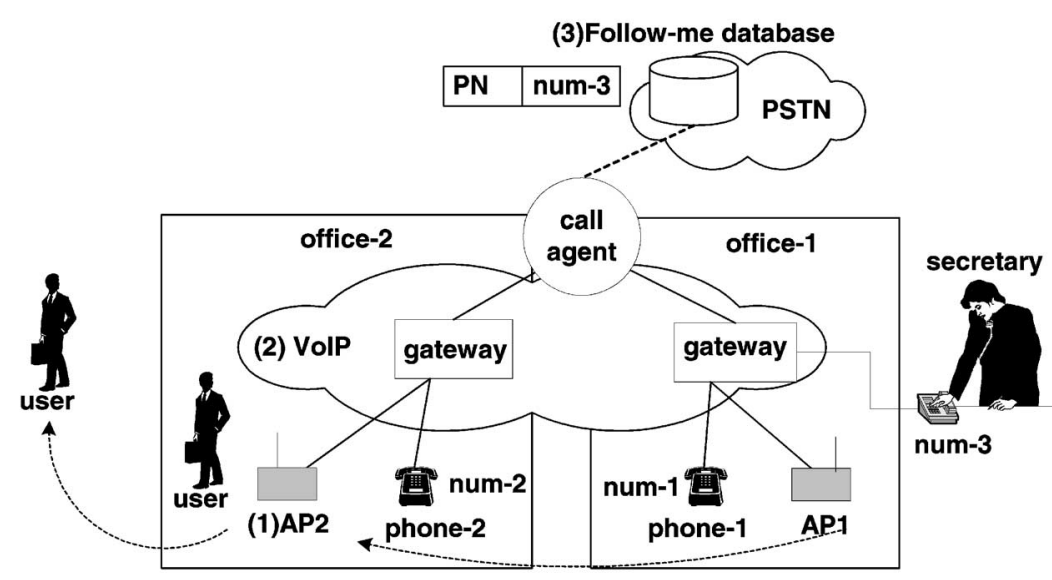

Fig. 2. Automatic Follow-me service: Scenario I. 


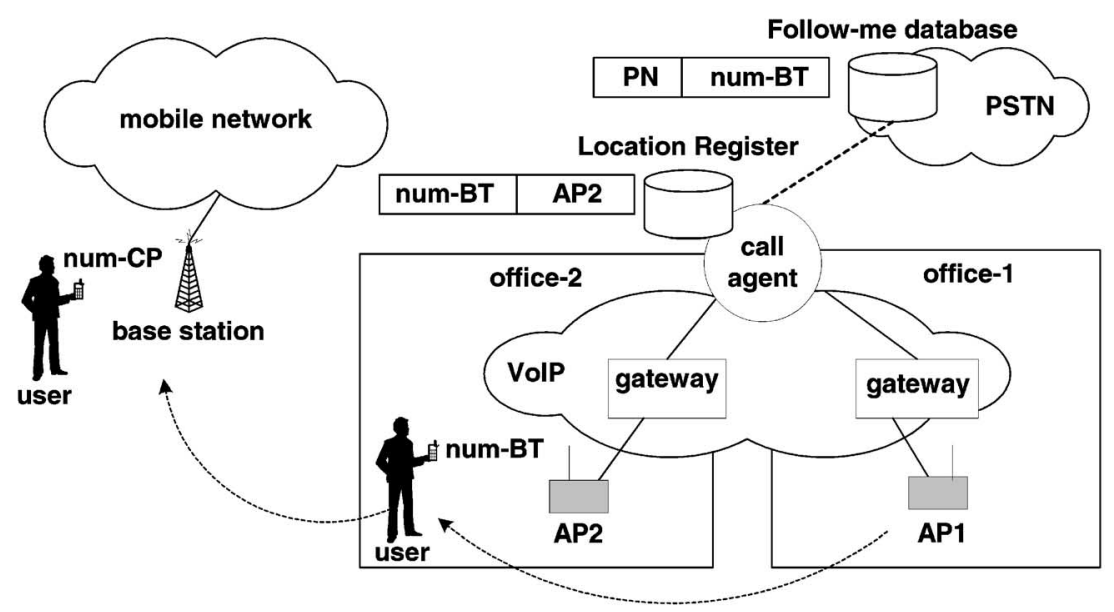

Fig. 3. Automatic Follow-me service: Scenario II.

It is clear that AFS is a useful enterprise service which can integrate with PN services offered by different PSTN operators. We show how AFS is implemented. Section 2 describes the AFS private telephony system implemented by using the VoIP technology. Section 3 shows the AFS radio tracking mechanism based on the Bluetooth technology. Finally, Section 4 analyzes the performance of the AFS polling mechanism.

\section{AFS VOIP NETWORK}

We utilize the Media Gateway Control Protocol (MGCP) in the AFS VoIP network. Based on the concept of gateway decomposition, MGCP [2] assumes a call control architecture where the call control "intelligence" is provided by call agents outside the telephony gateways. Specifically, MGCP standardizes the interfaces between the telephony gateways and call agents. Fig. 4 illustrates an MGCP-based VoIP system. In this figure, Media Gateway (MG) is a telephony gateway that provides conversion between the audio signals carried on the switched circuit network and data packets carried over the IP network. There are several MG types. Two types of MGs are used in AFS: Residential Gateway (RGW) and Trunking Gateway (TGW). The RGW provides a traditional analog interface which connects existing analog telephones and fax machines to the VoIP network. The TGW interfaces between the PSTN and a VoIP network. A TGW is typically a tandem switch connecting to a switch in the PSTN via T1 or E1 trunk. Signaling Gateway (SG) interworks the MGCP elements with the Signaling System Number 7 (SS7) signaling network [14] in the PSTN. This gateway performs conversion between the SS7 signaling protocols in the PSTN and the

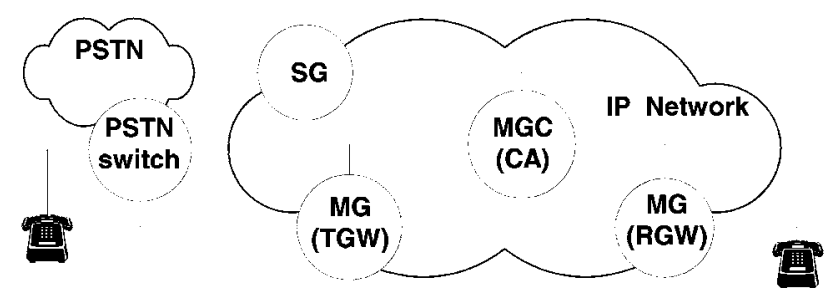

Fig. 4. MGCP architecture.
IETF Signaling Transport (SIGTRAN) protocol [16] in the IP network. It also maintains the address mapping function between the SS7 and IP addresses. Media Gateway Controller (MGC) or Call Agent (CA) is responsible for call setup and release for media channels in an MG. By utilizing the signaling protocol translation function in an SG, an MGC can handle SS7 signaling for call setup between IP network and PSTN.

Based on the MGCP architecture, we show how AFS registration, deregistration, and call delivery are implemented. The AFS architecture Scenario I (Fig. 2) is assumed. To accommodate AFS Scenario II, the procedures only need to be slightly modified. The AFS registration and deregistration procedures are illustrated in Fig. 5. When a user enters the coverage area of an access point AP1, the situation is detected by the radio tracking mechanism, to be described in Section 3. Then AP1 sends a Registration message to the RGW (Fig. 5(1)). Upon receipt of the Registration message, the RGW invokes the registration request procedure (Fig. 5(2)) to inform the CA about the PN and the telephone number to be registered. This procedure triggers the CA to execute the location update procedure (Fig. 5(3)). In this procedure, the CA updates the location information of the Follow-me database in the PSTN. The CA first connects to the PSTN following the same process as the manual Followme registration procedure except that the dialed digits are automatically generated. No new interface between the CA and the Follow-me database is introduced. Finally, the registration response procedure is executed to inform the AP1 that the AFS registration procedure has been completed (Fig. 5(4)). The deregistration procedure is shown in Steps (5)-(8) in Fig. 5. These steps are similar to those of the registration procedure except that, in the location update procedure (Fig. 5(7)), the PN record of the user in the Follow-me database is set to the default number (e.g., the cellular phone number of the user). Details of the MGCP message flows for registration and deregistration procedures are given in Appendix A.

When someone dials the PN number, the call setup procedure consists of two parts. The first part follows standard Follow-me database query (signaling path (d) in Fig. 6) and PSTN call setup to the AFS system (trunk path 


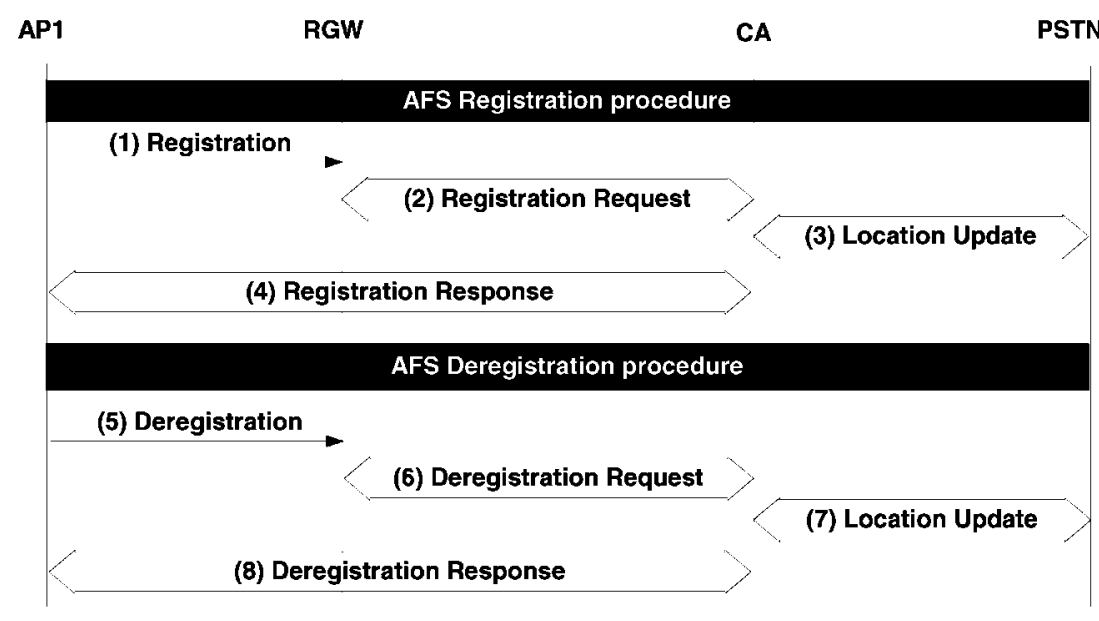

Fig. 5. The Simplified AFS registration and deregistration procedures.

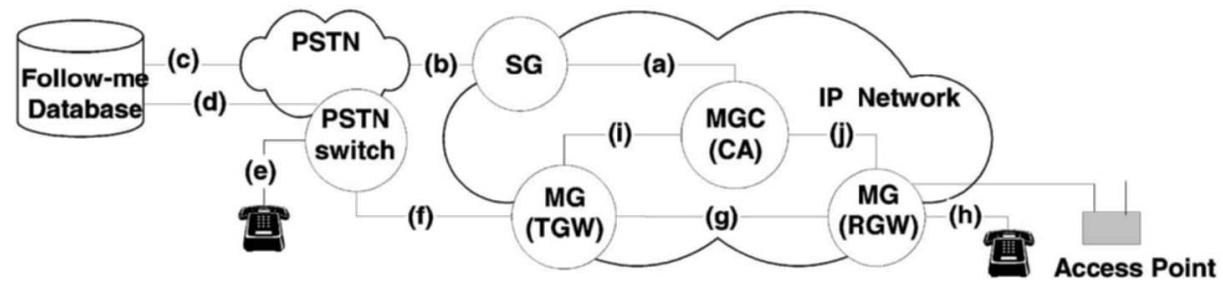

Fig. 6. AFS registration and call setup paths.

$(\mathrm{e}) \rightarrow$ (f) in Fig. 6 and signaling path (b) $\rightarrow$ (a) $\rightarrow$ (i)). In the second part, the CA utilizes the standard VoIP protocol to set up the call to the AP where the user resides (trunk path $(\mathrm{g}) \rightarrow(\mathrm{h})$ in Fig. 6 and signaling paths (i) and (j)). Details of VoIP call setup procedure can be found in [2]. This section shows that, through the MGCP call agent technology, we can use the existing PSTN interface to communicate with the Follow-me database. Therefore, with our approach, a telephone company does not need to modify the existing Follow-me service. In the current stage, we have designed a development toolkit to trace the events and messages of the above AFS procedures. Through this tool, we have tested many scenarios which showed that our procedures work correctly. Fig. 7 illustrates the graphical user interface of the development toolkit.

\section{AFS Radio Tracking System}

AFS utilizes the Bluetooth wireless technology [3] for wireless access and $\mathrm{PN}$ user tracking. Bluetooth is a standard for short-range radio links between portable devices (PDs) such as mobile PCs and mobile phones. Several Bluetooth access points (APs) are populated to provide complete radio coverage in an enterprise AFS environment. Each of the APs is the master of a piconet, which detects the slaves (i.e., PDs) entering the piconet. In this piconet, the master is responsible for initiating the connection to the slaves by giving slaves its clock and device identifier (ID). Fig. 8 illustrates the architecture of the Bluetooth device, which consists of two parts: the Host and the Bluetooth module. The Host controls the Bluetooth module via the Host Controller Interface (HCI). In the Host, the application (Fig. 8(1)) issues commands or data to the
HCI layer (Fig. 8(2)). The HCI layer exercises the HCI communications protocol to deliver data to and from the Bluetooth module through the Host Controller (Fig. 8). In the Bluetooth module, the Link Manager (Fig. 8(4)) supports functions for security management, power management, and QoS management. The Link Controller (Fig. 8(5)) provides baseband functions such as connection establishment and frequency selection. The Radio module (Fig. 8(6)) is responsible for radio transmission. We utilize the Bluetooth Link Controller functions and Link Manager functions to implement the Bluetooth registration and deregistration procedures. Fig. 9 illustrates the registration and deregistration procedures executed between the AP and the PD, which are described as follows:

Step 1: PD Detection. Through the Bluetooth inquiry procedure, the AP periodically checks if any new PDs have moved into the service area and the PD listens to the broadcast of the AP. The PD responds to the AP when it receives the signal from the AP.

Step 2: Signaling Path Establishment. If the AP discovers a PD through the inquiry procedure, a connection between the AP and the PD is established through the Bluetooth page procedure.

Step 3: Registration. The AP invokes the AFS registration procedure (described in Section 2) to update the PD's PN record in the Follow-me database.

Step 4: Power Saving. To save power, the Bluetooth park procedure is executed to instruct the PD to operate in the low power-consumption mode. 


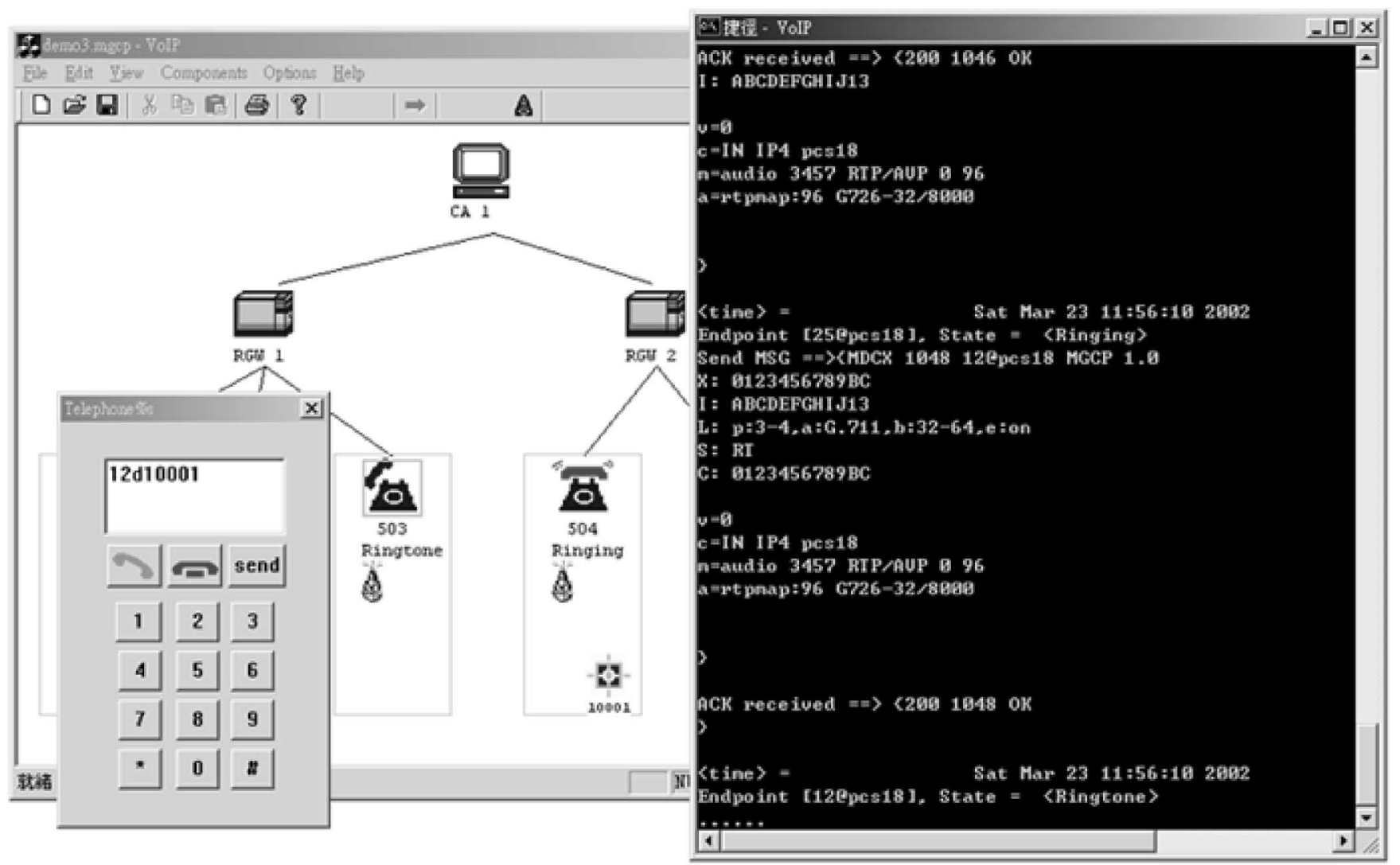

Fig. 7. The graphical user interface of our VolP development toolkit. (The screen shows the scenario that a user moves from AP 503 to AP 504 . The window on the right prints out the VolP messages for the AFS procedures.)

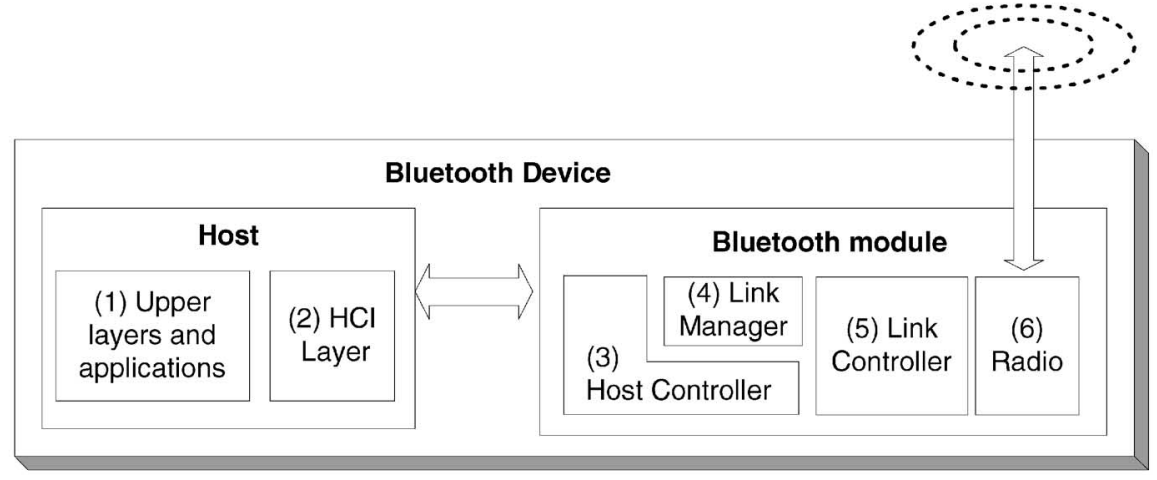

Fig. 8. Bluetooth device architecture.

\section{Step 5: Subsequent Polling. After registration, both the AP} and the PD are connected. The AP periodically polls the PD to check if the PD is still in the service area.

Step 6: Deregistration. When the PD leaves the coverage area of the AP, the situation will be detected by the AP through periodic polling described in Step 5. The AP then invokes the AFS deregistration procedure (see Section 3) to update the PD's PN record in the Followme database.

The implementation details (specifically, the inquiry, page, park, and other related Bluetooth procedures) are given in Appendix B. Fig. 10 illustrates the AFS tracking prototype.

\section{Performance of the Polling Mechanism}

Several performance issues are considered for the AFS polling mechanism to achieve efficient energy management for PD [20] as well as to reduce misrouting. We elaborate on these issues as follows:

Polling Interval Distribution. Most polling mechanisms utilize fixed-length polling intervals. In the AFS Bluetooth tracking system, the AP detects the arrivals of PDs through periodic broadcast of inquiry messages. Thus, all PDs arriving between two inquiry broadcasts will be detected at the same time. If fixed-length polling interval is used, then subsequent pollings for these PDs will occur at the same times. To avoid "polling congestion," we consider exponential polling intervals (just like the 


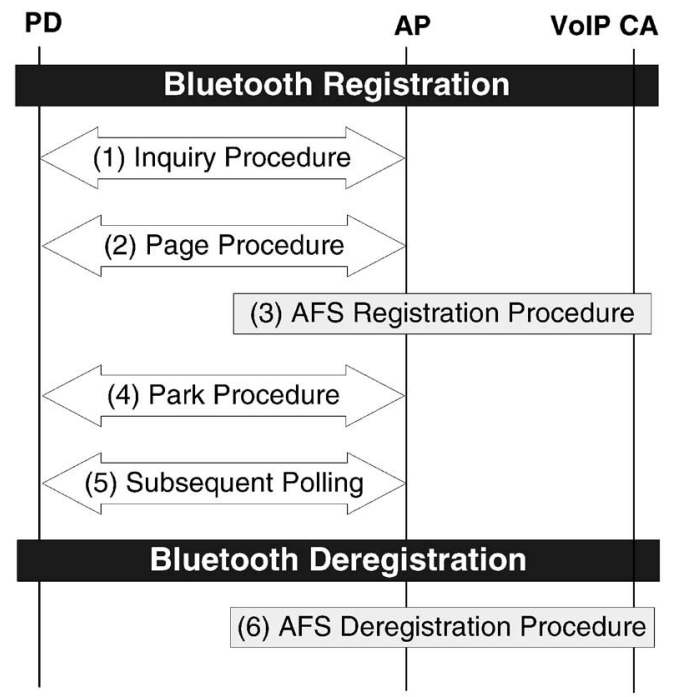

Fig. 9. The simplified Bluetooth registration and deregistration procedures.

exponential backup retry strategy used in the slotted Aloha network). With this arrangement, although the mean polling intervals are the same, the PDs are polled at different times to avoid congestion. Without considering the polling congestion effect (i.e., the optimal case for fixed polling intervals), our simulation experiments indicate similar performance for both fixed and exponential polling intervals (not presented in this paper). Discussion of the impact of registration frequency on message collisions can be found in [9].

Polling Frequency. If polling is not frequent enough, the AP may not detect the absence of a PD early and some incoming calls may be misrouted. On the other hand, if polling is too frequent, the PD may consume too much power. Thus, it is important to select an appropriate frequency that balances against power consumption and call misrouting.

The number of pollings can also be reduced if the user movement patterns are known. For example, if we know that, on the average, a specific user stays in the AFS area for three hours, then the first polling interval can be set to a long period, say, 1.5 hours. Then, the subsequent pollings are conducted at a much higher frequency (say, 5 minutes per polling).

This section proposes an analytic model to investigate the effect of polling frequency. Note that our polling frequency analysis also applies to general Bluetooth location applications. However, this analysis is particularly important for AFS because AFS is much more sensitive to location accuracy than other telecommunication services. In Follow-me service, incorrect location information leads to misrouted calls that may be answered by the wrong person, which causes privacy issues. For general mobile telecommunication services, networks without correct location

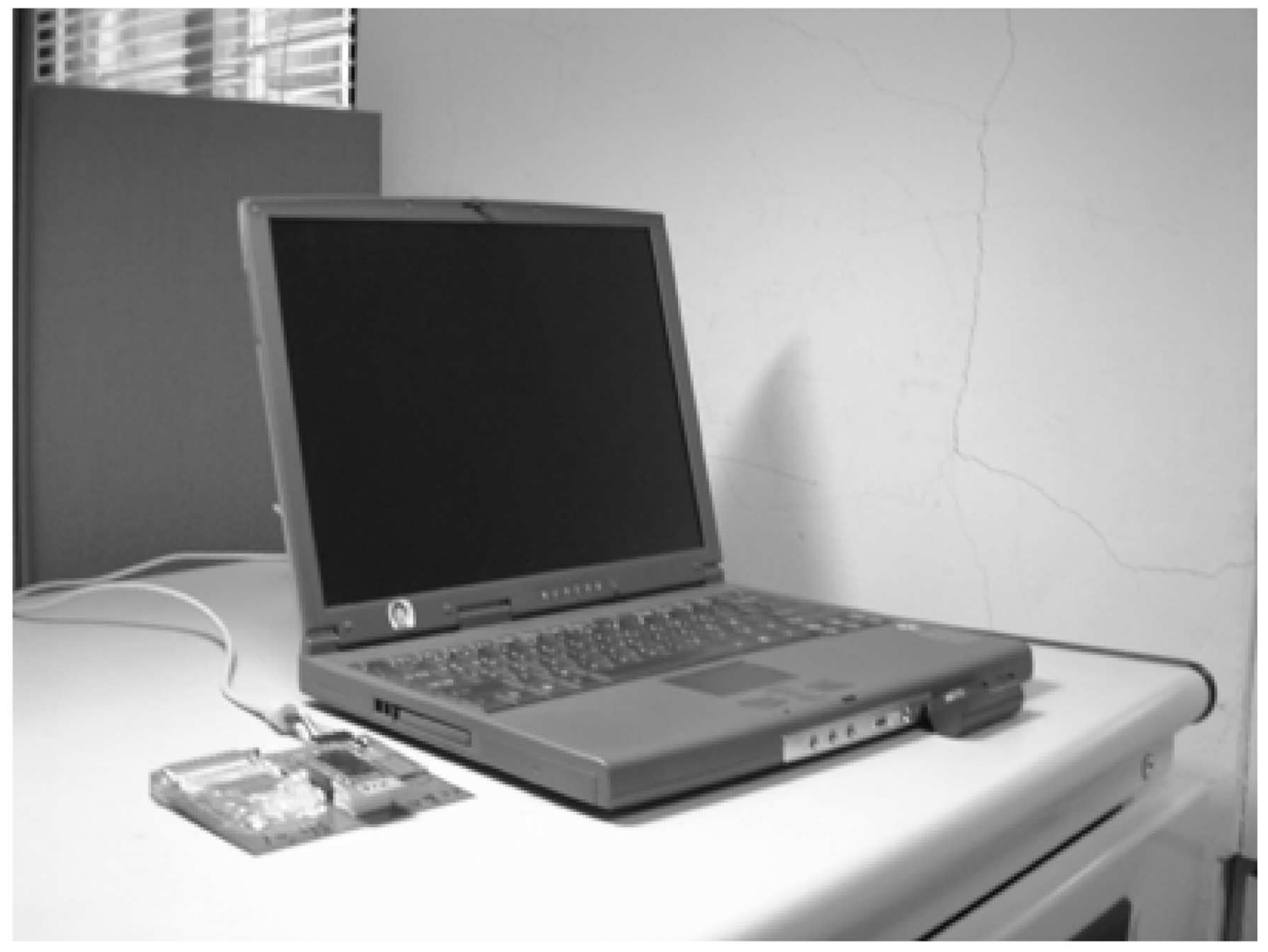

Fig. 10. AFS Bluetooth tracking prototype. (The notebook is the AP controller that connected to the VolP system. The circuit board on the left of the notebook is the Bluetooth AP module.) 
information will drop the calls or forward them to voice mailboxes. Therefore, our analysis is very useful for AFS parameter setup. In our study, output measures are the number of misrouted calls and the number of pollings (and thus power consumption). Consider the timing diagram in Fig. 11c. Let random variable $t_{m}$ represent the period when the user resides in the AFS service area, which has the density function $f_{m}\left(t_{m}\right)$, the expected value $1 / \eta$, and the Laplace transform $f_{m}^{*}(s)=\int_{t=0}^{\infty} f_{m}(t) e^{-s t} d t$. Let random variable $\tau_{i}$ (where $i=0,1,2, \ldots$ ) represent the period between the $i$ th and the $i+1$ st pollings, which has exponential density function $f_{i}\left(\tau_{i}\right)=\mu_{i} e^{-\mu_{i} \tau_{i}}$ with rate $\mu_{i}$. Note that the zeroth polling represents the inquiry broadcast of the AP, which detects the existence of the PD. As mentioned earlier, $\tau_{0}$ is selected based on $E\left[t_{m}\right]$ computed from the previous measures. To be conservative, a fudge factor $\alpha<1$ is used to make sure that the first polling occurs before the user leaves the AFS service area. That is,

$$
E\left[\tau_{0}\right]=\alpha E\left[t_{m}\right] \quad \text { or } \quad \mu_{0}=\frac{\eta}{\alpha} .
$$

Consider the example where past experience indicates that, on the average, the user will stay in the area for $\frac{1}{\eta}=3$ hours and we set the fudge factor $\alpha=0.5$. That is, the user is expected to stay longer than $\frac{\alpha}{\eta}=1.5$ hours and the AFS will perform the first polling based on the density function $f_{0}\left(\tau_{0}\right)$ with mean 1.5 hours. After the first polling, the subsequent polling rate $\mu_{i}(i>0)$ is significantly increased; that is, $\mu_{i}=\mu>>\mu_{0}$ for $i>0$. For derivation purposes, let $\mu_{0}=\beta \mu$. From (1), we have $\frac{\eta}{\alpha}=\beta \mu$ or $\beta=\frac{\eta}{\alpha \mu}$. Let random variable $t_{k}$ be the interval between the first polling and the $k+1$ st polling, then $t_{k}=\tau_{1}+\tau_{2}+\ldots+\tau_{k}$ has Erlang-k distribution with the density function $f_{p, k}\left(t_{k}\right)$, where

$$
f_{p, k}\left(t_{k}\right)=\left[\frac{\left(\mu t_{k}\right)^{k-1}}{(k-1) !}\right] \mu e^{-\mu t_{k}} .
$$

Let random variable $t_{l}$ be the period between when the user leaves the AFS service area and when the next polling occurs. The density function $f_{l}\left(t_{l}\right)$ of $t_{l}$ is derived in three cases:

Case I $\left(\tau_{0}>t_{m}\right)$. There is one polling before the PD leaves the AFS area (see Fig. 11a). Note that any PD entering the AFS area will experience at least one polling (i.e., the zeroth polling due to the AP inquiry). In this case, let $f_{l, I}\left(t_{l}\right)$ be the density function of $t_{l}$. We have $\tau_{0}=t_{m}+t_{l}$ and

$$
\begin{aligned}
f_{l, I}\left(t_{l}\right) & =\int_{t_{m}=0}^{\infty} f_{m}\left(t_{m}\right) f_{0}\left(t_{m}+t_{l}\right) d t_{m} \\
& =\beta \mu e^{-\beta \mu t_{l}} f_{m}^{*}(\beta \mu) .
\end{aligned}
$$

Case II ( $\left.\tau_{0} \leq t_{m} \leq \tau_{0}+\tau_{1}\right)$. There are two pollings before the PD leaves the AFS area (see Fig. 11b). In this case, let $f_{l, I I}\left(t_{l}\right)$ be the density function of $t_{l}$. We have $\tau_{1}=$ $t_{m}+t_{l}-\tau_{0}$ and

$$
\begin{aligned}
& f_{l, I I}\left(t_{l}\right) \\
& =\int_{t_{m}=0}^{\infty} \int_{\tau_{0}=0}^{t_{m}} f_{m}\left(t_{m}\right) f_{0}\left(\tau_{0}\right) f_{1}\left(t_{m}+t_{l}-\tau_{0}\right) d \tau_{0} d t_{m} \\
& =\left(\frac{\beta \mu e^{-\mu t_{l}}}{\beta-1}\right) \int_{t_{m}=0}^{\infty} f_{m}\left(t_{m}\right)\left(e^{-\mu t_{m}}-e^{-\beta \mu t_{m}}\right) d t_{m} \\
& =\left(\frac{\beta \mu e^{-\mu t_{l}}}{\beta-1}\right)\left[f_{m}^{*}(\mu)-f_{m}^{*}(\beta \mu)\right] .
\end{aligned}
$$

Case III $\left(\tau_{0}+t_{k} \leq t_{m}<\tau_{0}+t_{k}+\tau_{k+1}\right.$ for $\left.k \geq 1\right)$. There are $k+2$ pollings before the PD leaves the AFS area (see Fig. 11c). In this case, let $f_{l, I I I, k}\left(t_{l}\right)$ be the density function of $t_{l}$. We have $\tau_{k+1}=t_{m}+t_{l}-\tau_{0}-t_{k}$ and

$$
\begin{aligned}
& f_{l, I I I, k}\left(t_{l}\right) \\
& =\int_{t_{m}=0}^{\infty} \int_{t_{k}=0}^{t_{m}} \int_{\tau_{0}=0}^{t_{m}-t_{k}} f_{m}\left(t_{m}\right) f_{p, k}\left(t_{k}\right) \\
& \quad \times f_{0}\left(\tau_{0}\right) f_{k+1}\left(t_{m}+t_{l}-\tau_{0}-t_{k}\right) d \tau_{0} d t_{k} d t_{m} \\
& =\left(\frac{\beta \mu}{\beta-1}\right) e^{-\mu t_{l}} \int_{t_{m}=0}^{\infty} f_{m}\left(t_{m}\right) e^{-\mu t_{m}} \\
& \quad \times \int_{t_{k}=0}^{t_{m}} f_{p, k}\left(t_{k}\right) e^{\mu t_{k}}\left[1-e^{-(\beta-1) \mu\left(t_{m}-t_{k}\right)}\right] d t_{k} d t_{m} \\
& =\left(\frac{\beta \mu}{\beta-1}\right) e^{-\mu t_{l}}(A-B),
\end{aligned}
$$

where, from (2)

$$
\begin{aligned}
A & =\int_{t_{m}=0}^{\infty} f_{m}\left(t_{m}\right) e^{-\mu t_{m}}\left[\int_{t_{k}=0}^{t_{m}} f_{p, k}\left(t_{k}\right) e^{\mu t_{k}} d t_{k}\right] d t_{m} \\
& =\int_{t_{m}=0}^{\infty} f_{m}\left(t_{m}\right) e^{-\mu t_{m}}\left[\frac{\left(\mu t_{m}\right)^{k}}{k !}\right] d t_{m} \\
& =\left.\left[\frac{(-\mu)^{k}}{k !}\right]\left[\frac{d^{k} f_{m}^{*}(s)}{d s^{k}}\right]\right|_{s=\mu}
\end{aligned}
$$

and

$$
\begin{aligned}
B= & \int_{t_{m}=0}^{\infty} f_{m}\left(t_{m}\right) e^{-\mu t_{m}} \\
& \times\left[\int_{t_{k}=0}^{t_{m}} f_{p, k}\left(t_{k}\right) e^{\mu t_{k}} e^{-(\beta-1) \mu\left(t_{m}-t_{k}\right)} d t_{k}\right] d t_{m} \\
= & \int_{t_{m}=0}^{\infty} f_{m}\left(t_{m}\right) e^{-\beta \mu t_{m}}\left(\frac{1}{1-\beta}\right)^{k} \\
& \times\left\{1-\sum_{j=0}^{k-1}\left\{\frac{\left[(1-\beta) \mu t_{m}\right]^{j}}{j !}\right\} e^{-(1-\beta) \mu t_{m}}\right\} d t_{m} \\
= & \left.\frac{1}{1-\beta}\right)^{k}\left\{f_{m}^{*}(\beta \mu)-\sum_{j=0}^{k-1}\left\{\frac{[(1-\beta) \mu]^{j}}{j !}\right\}\right. \\
& \left.\times \int_{t_{m}=0}^{\infty} t_{m}^{j} f_{m}\left(t_{m}\right) e^{-\mu t_{m}} d t_{m}\right\} \\
= & \left.\frac{1}{1-\beta}\right)^{k}\left\{f_{m}^{*}(\beta \mu)\right. \\
& \left.-\left.\sum_{j=0}^{k-1}\left\{\frac{[(\beta-1) \mu]^{j}}{j !}\right\}\left[\frac{d^{j} f_{m}^{*}(s)}{d s^{j}}\right]\right|_{s=\mu}\right\} .
\end{aligned}
$$

From (5), (6), and (7), we have 


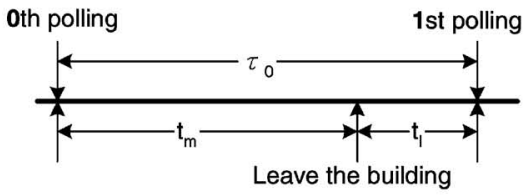

(a)

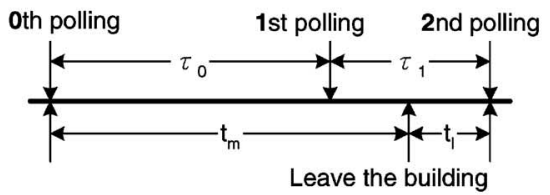

(b)

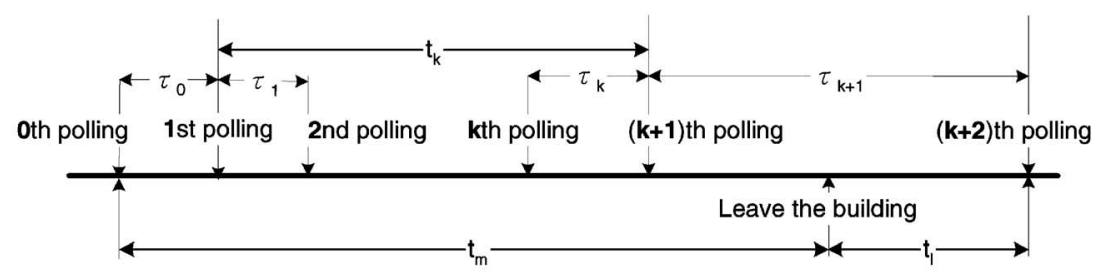

(c)

Fig. 11. The timing diagram.

$$
f_{l, I I I, k}\left(t_{l}\right)=\left(\frac{\beta \mu e^{-\mu t_{l}}}{\beta-1}\right) X_{k},
$$

where, for $k \geq 1$,

$$
\begin{aligned}
& X_{k}=\left.\left[\frac{(-\mu)^{k}}{k !}\right]\left[\frac{d^{k} f_{m}^{*}(s)}{d s^{k}}\right]\right|_{s=\mu} \\
&-\left(\frac{1}{1-\beta}\right)^{k}\left\{f_{m}^{*}(\beta \mu)\right. \\
&\left.-\left.\sum_{j=0}^{k-1}\left\{\frac{[(\beta-1) \mu]^{j}}{j !}\right\}\left[\frac{d^{j} f_{m}^{*}(s)}{d s^{j}}\right]\right|_{s=\mu}\right\} .
\end{aligned}
$$

From (3), (4), and (8), we have

$$
\begin{aligned}
f_{l}\left(t_{l}\right) & =f_{I, l}\left(t_{l}\right)+f_{I I, l}\left(t_{l}\right)+\sum_{k=1}^{\infty} f_{I I I, l, k}\left(t_{l}\right) \\
& =C_{1} e^{-\beta \mu t_{l}}+C_{2} e^{-\mu t_{l}},
\end{aligned}
$$

where

$$
\begin{aligned}
C_{1} & =\beta \mu f_{m}^{*}(\beta \mu) \text { and } \\
C_{2} & =\left(\frac{\beta \mu}{\beta-1}\right)\left[f_{m}^{*}(\mu)-f_{m}^{*}(\beta \mu)+\sum_{k=1}^{\infty} X_{k}\right] .
\end{aligned}
$$

Suppose that the call arrivals to a mobile user are a Poisson stream with rate $\lambda$. Then, from (11), the probability of $n$ misrouted calls is

$$
\begin{aligned}
\operatorname{Pr}[N=n] & =\int_{t_{l}=0}^{\infty}\left[\frac{\left(\lambda t_{l}\right)^{n}}{n !}\right] e^{-\lambda t_{l}} f_{l}\left(t_{l}\right) d t_{l} \\
& =\frac{C_{1} \lambda^{n}}{(\lambda+\beta \mu)^{n+1}}+\frac{C_{2} \lambda^{n}}{(\lambda+\mu)^{n+1}} .
\end{aligned}
$$

From (12), the expected number $E[N]$ of misrouted calls is

$$
E[N]=\sum_{n=1}^{\infty} n \operatorname{Pr}[N=n]=\left[\frac{C_{1}}{(\beta \mu)^{2}}+\frac{C_{2}}{\mu^{2}}\right] \lambda .
$$

Let $K$ be the number of pollings including the AP inquiry (i.e., the zeroth polling). Then,

$$
\begin{gathered}
\operatorname{Pr}[K=1]=\operatorname{Pr}\left[\tau_{0}>t_{m}\right] \\
=\int_{t_{l}=0}^{\infty} f_{l, I}\left(t_{l}\right) d t_{l} \\
=f_{m}^{*}(\beta \mu) \\
\operatorname{Pr}[K=2]=\operatorname{Pr}\left[\tau_{0}<t_{m}<\tau_{0}+\tau_{1}\right] \\
=\int_{t_{l}=0}^{\infty} f_{l, I I}\left(t_{l}\right) d t_{l} \\
=\left(\frac{\beta}{\beta-1}\right)\left[f_{m}^{*}(\mu)-f_{m}^{*}(\beta \mu)\right] .
\end{gathered}
$$

For $k \geq 1$,

$$
\begin{aligned}
\operatorname{Pr}[K=k+2] & =\operatorname{Pr}\left[\tau_{0}+t_{k}<t_{m}<\tau_{0}+t_{k}+\tau_{k+1}\right] \\
& =\int_{t_{l}=0}^{\infty} f_{l, I I I}\left(t_{l}\right) d t_{l} \\
& =\left(\frac{\beta}{\beta-1}\right) X_{k} .
\end{aligned}
$$

From (14), (15), and (16), the expected number $E[K]$ of

pollings is

$$
\begin{aligned}
E[K]= & \sum_{k=1}^{\infty} k \operatorname{Pr}[K=k] \\
= & \left(\frac{1}{\beta-1}\right)\left[2 \beta f_{m}^{*}(\mu)\right. \\
& \left.-(1+\beta) f_{m}^{*}(\beta \mu)+\sum_{k=1}^{\infty}(k+2) \beta X_{k}\right] .
\end{aligned}
$$

If $f_{m}\left(t_{m}\right)$ is Erlang-M distributed, then

$$
f_{m}^{*}(s)=(M \eta)^{M} /(M \eta+s)^{M} .
$$

From (9), (11), and (18), we have

$$
C_{1}=\frac{\beta \mu(M \eta)^{M}}{(M \eta+\beta \mu)^{M}}
$$



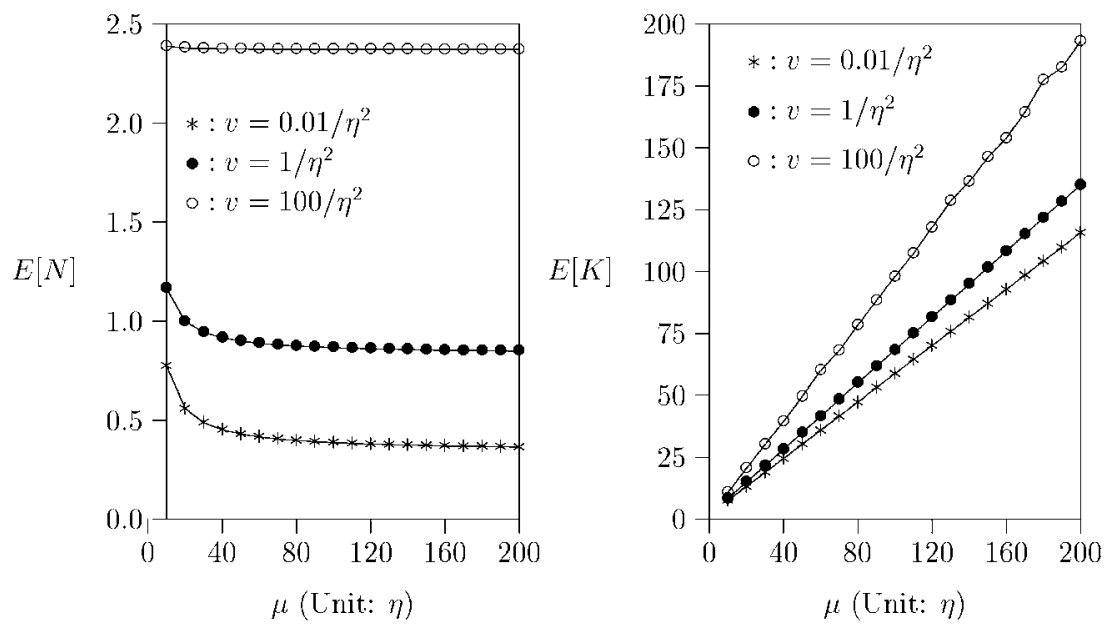

Fig. 12. Effects of $v(\alpha=0.5, \lambda=5 \eta$; both $E[N]$ and $E[K]$ are normalized by $\eta)$.

TABLE 1

Analytic Analysis vs. Simulation Results $(\alpha=0.5, \lambda=5 \eta$; Both $E[N]$ and $E[K]$ Are Normalized by $\eta)$

\begin{tabular}{|l||l|l||l|l||l|l|}
\hline$\mu$ & $10 \eta($ Ana) & $10 \eta(\mathrm{Sim})$ & $100 \eta($ Ana) & $100 \eta(\mathrm{Sim})$ & $500 \eta$ (Ana) & $500 \eta$ (Sim) \\
\hline$E[N]\left(v=0.01 / \eta^{2}\right)$ & 0.7761 & 0.7557 & 0.3882 & 0.3949 & 0.3537 & 0.3471 \\
\hline$E[N]\left(v=1 / \eta^{2}\right)$ & 1.1667 & 1.1552 & 0.8667 & 0.8673 & 0.84 & 0.8553 \\
\hline$E[N]\left(v=100 / \eta^{2}\right)$ & 2.3884 & 2.4244 & 2.3727 & 2.3685 & 2.3712 & 2.353 \\
\hline
\end{tabular}

$$
\begin{aligned}
C_{2}= & \left(\frac{\beta \mu}{\beta-1}\right)\left[\left(\frac{M \eta}{M \eta+\mu}\right)^{M}\right. \\
& \left.-\left(\frac{M \eta}{M \eta+\beta \mu}\right)^{M}+\sum_{k=1}^{\infty} X_{k}\right]
\end{aligned}
$$

$$
\begin{aligned}
X_{k}= & {\left[\frac{1}{(1-\beta)^{k}}\right]\left\{( \frac { M \eta } { M \eta + \mu } ) ^ { M } \left\{\sum_{j=0}^{k}\left(\begin{array}{c}
M+j-1 \\
j
\end{array}\right)\right.\right.} \\
& \left.\left.\times\left[\frac{\mu(1-\beta)}{M \eta+\mu}\right]^{j}\right\}-\left(\frac{M \eta}{M \eta+\beta \mu}\right)^{M}\right\} .
\end{aligned}
$$

Note that (19)-(21) can be easily extended for the $t_{m}$ with the hyper-Erlang distribution [6], [11]. This distribution has a very general approximation capability to the probability distribution of any nonnegative random variable. For $M=1$ :

$$
C_{1}=\frac{\beta \mu \eta}{\eta+\beta \mu}, \quad C_{2}=\left[\frac{\beta \mu(\eta+\mu)}{\beta-1}\right]\left(\frac{1}{\eta+\mu}-\frac{1}{\eta+\beta \mu}\right) .
$$

Based on the analytic model, we show how the polling frequency and the user movement behavior affect the PD power consumption and call misrouting. The analytic results are validated against the simulation experiments. The simulation model is similar to the one developed in [13]. The details are omitted. We consider the Gamma $t_{m}$ distribution. The Laplace Transform of Gamma distribution is the same as that in (18) except that the parameter $M$ is replaced by a positive real number. The distribution of any positive random variable can be approximated by a mixture of Gamma distributions (see Lemma 3.9 in [11]). One may also measure the $t_{m}$ values in the AFS network and the measured data can be approximated by a Gamma distribution [4], [7], [8], [18] as the input to our analytic model. The results for the hyper-Erlang distribution are similar to the Gamma distribution and are not presented in this paper.

From (13), it is clear that $E[N]$ is a linear function of the call arrival rate $\lambda$. Fig. 12 plots $E[N]$ and $E[K]$ against the variance $v$ for the AFS residence times of a PD (where $\alpha=0.5, \lambda=5 \eta$ ). Table 1 lists some data points in Fig. 12 for both analytic analysis and simulation experiments. The table indicates that the analytic results (labeled "Ana") are consistent with the simulation results (labeled "Sim"). Fig. 13 plots $E[N]$ and $E[K]$ against $\alpha$, where $\lambda=5 \eta$ and $v=1 / \eta^{2}$.

It is apparent that $E[N]$ decreases as $\mu$ increases. As shown in Figs. 12 and 13, there exists a threshold $\mu^{*}$ such that, when $\mu>\mu^{*}$, increasing $\mu$ does not improve the $E[N]$ performance. Fig. 12 indicates that, as $v$ increases, $\mu^{*}$ becomes smaller. On the other hand, $E[K]$ constantly increases as $\mu$ increases. Our experiments indicate that it is appropriate to select $100 \eta \leq \mu^{*} \leq 150 \eta$. As an example, consider the " $\circ$ " curves in Fig. 13. By increasing $\mu$ from $10 \eta$ to $150 \eta, E[N]$ is improved by 98 percent. On the other hand, if $\mu$ is increased from $150 \eta$ to $200 \eta, E[N]$ is only improved by 13 percent. Fig. 12 shows that $E[N]$ increases as $v$ increases. As $v$ increases, more short and long $t_{m}$ intervals are observed in the system and Case I in Fig. 11a is more likely to occur. Furthermore, it is more likely to observe long $t_{l}$ in Case I for large $v$. Thus, large $E[N]$ is expected. Similarly, $E[K]$ increases as $v$ increases.

Figs. 13 and 14 illustrate the effect of $\alpha$. It is clear that, as $\alpha$ increases, $E[N]$ increases and $E[K]$ decreases. When the variance of $t_{m}$ is large (see Fig. 13), although $E[K]$ can be effectively reduced by a large $\alpha$ value, the side effect of increasing $E[N]$ cannot be ignored. In a typical enterprise environment, the working hour patterns of employees are more regular and the variance of $t_{m}$ tends to be small. In 

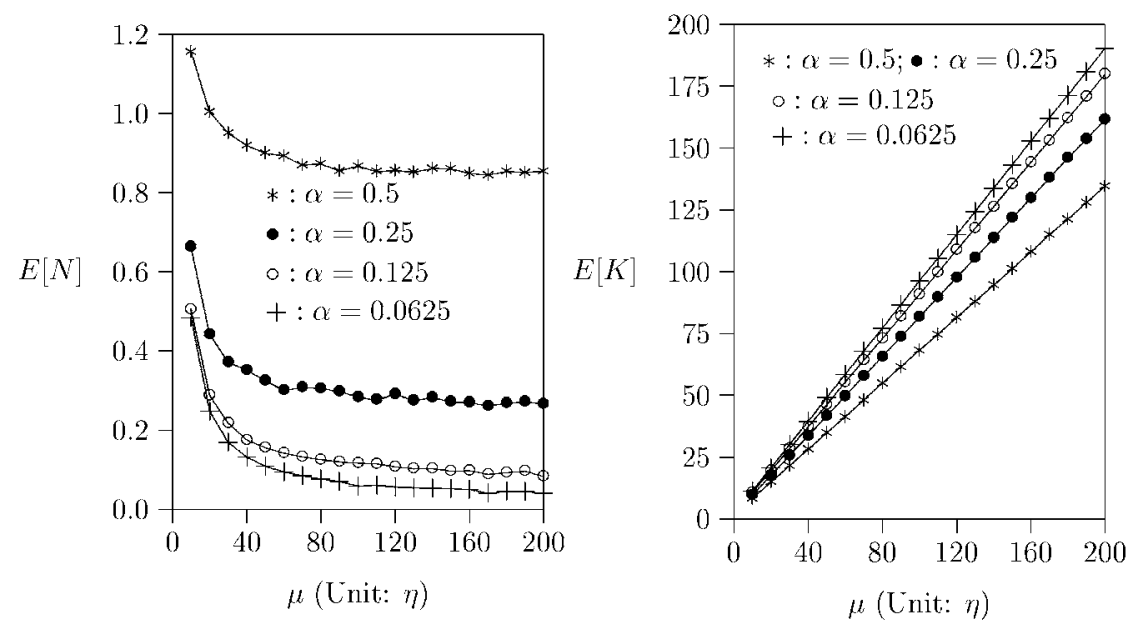

Fig. 13. Effects of $\alpha\left(\lambda=5 \eta, v=1 / \eta^{2}\right.$; both $E[N]$ and $E[K]$ are normalized by $\left.\eta\right)$.
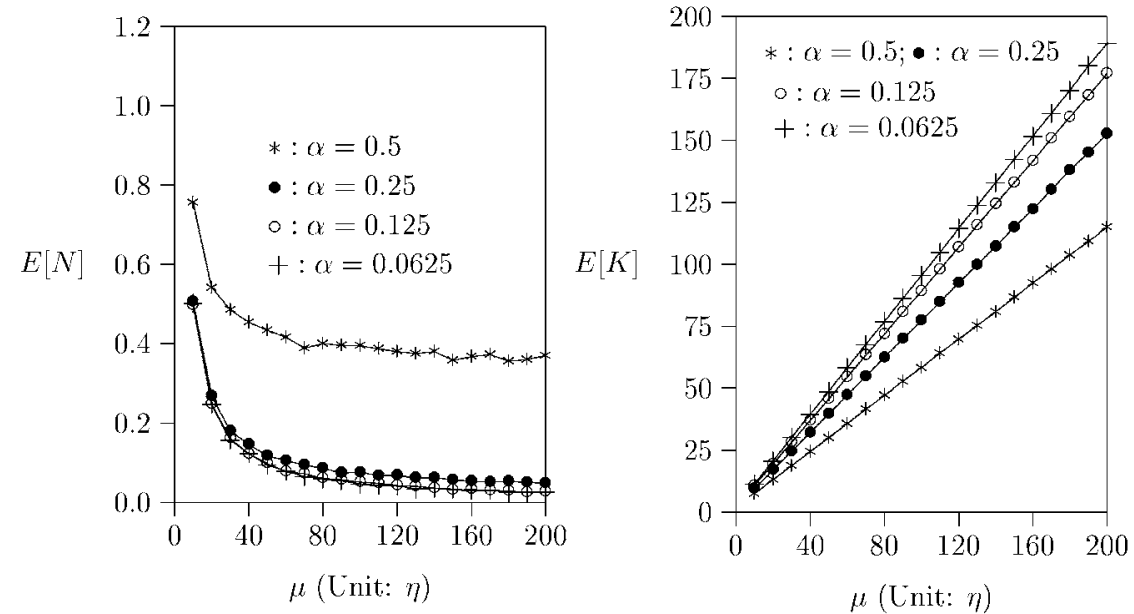

Fig. 14. Effects of $\alpha\left(\lambda=5 \eta, v=0.01 / \eta^{2}\right.$; both $E[N]$ and $E[K]$ are normalized by $\left.\eta\right)$.

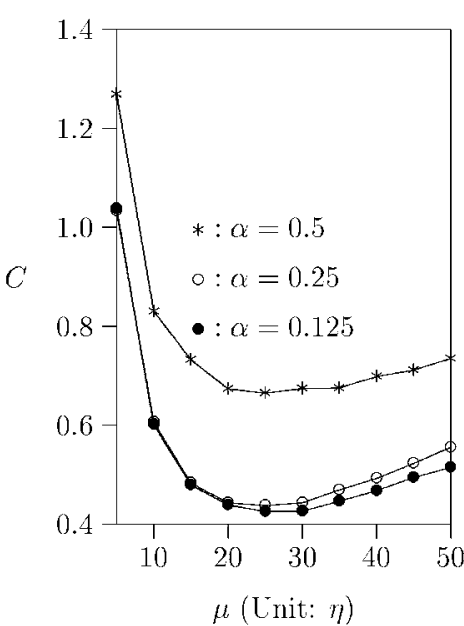

(a)

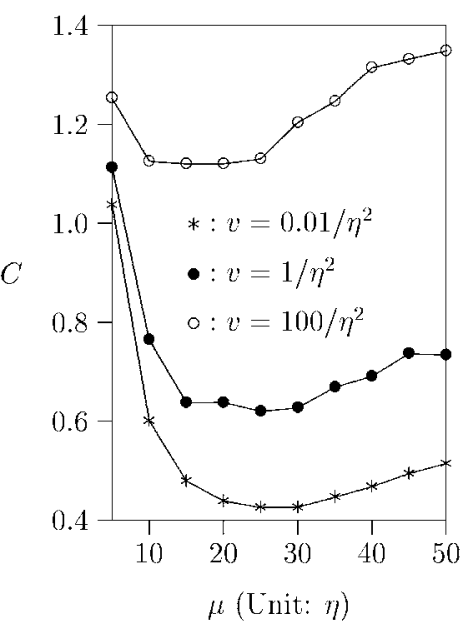

(b)

Fig. 15. Effects of polling on the cost $C(\lambda=5 \eta, \gamma=0.01)$.

this case, an appropriate $\alpha$ can effectively reduce $E[K]$ without increasing $E[N]$ (i.e., $\alpha=0.25$ Fig. 14).

One may use a cost function to select the optimal polling frequency. For example, consider the following simple cost function:

$$
C=E[N]+\gamma E[K],
$$

where $\gamma$ is a cost factor. Based on (23), Fig. 15 plots the cost $C$ against the polling frequency $\mu$, where $\gamma=0.01$. Fig. 15 shows that, for various $\alpha$ values, the optimal polling 
frequency is in the range $20 \eta \leq \mu \leq 30 \eta$. Fig. 15a shows that $\alpha=0.25$ is an appropriate selection that minimize the cost $C$. Fig. 15b shows that similar trends for $C$ are observed for various variances of the Gamma AFS residence time distribution.

\section{Conclusions}

This paper proposed an enterprise approach for automatic Follow-me service (AFS). The significance of our approach is that AFS can be integrated with existing Follow-me databases to automate PN services offered by different PSTN service providers. AFS automatically connects calls to a user at any location with appropriate communication terminals. We showed how to implement AFS with the VoIP and the Bluetooth technologies. Specifically, the AFS utilizes VoIP to communicate with the Follow-me database in the public network and Bluetooth is used to implement radio tracking mechanism. We then investigated the impact of polling frequency on power consumption and call misrouting. Our analysis guarantees that, based on the cost function of the AFS, the optimal polling frequency can be efficiently found. One of our future extensions is to develop automatic polling frequency adjustment heuristics based on our analytic model.

\section{APPENDIX A}

\section{The MGCP Registration and Deregistration Procedures}

The AFS location update mechanism follows the same concept for mobile networks (see [1], [14], [12] and the references therein). The AFS CA maintains a finite state machine with the state diagram shown in Fig. 16. In this diagram, the call model (the black box in Fig. 16) is a standard VoIP call model [2]. To support AFS registration and deregistration procedures, we introduce the Registration state, the Deregistration state, and the Location Update state. Transitions among the states are triggered by the MGCP commands. Every MGCP command is accompanied with an acknowledgment. The acknowledgment can be piggybacked with another MGCP command, which will not be shown in this paper. Fig. 17 illustrates the message flows for AFS registration and deregistration procedures with the following steps:

Step 1. Initially, the CA is in the Null state. When the AFS RGW starts up, it informs the CA of its presence by sending a Restart In Progress command. Then, the CA sends a Notification Request command to the RGW, which requests the RGW to detect the off-hook event. This step initializes the CA and the RGW for registration handling. After this step, the CA remains in the Null state (Transition 1 in Fig. 16).

Step 2. When a user enters the coverage area of an access point AP1, the situation is detected by the radio tracking mechanism described in Section 3. Then, AP1 sends a Registration message to the RGW. The Registration message contains the PN of the user and the endpoint ID. We assume that every AP is associated with a fixed

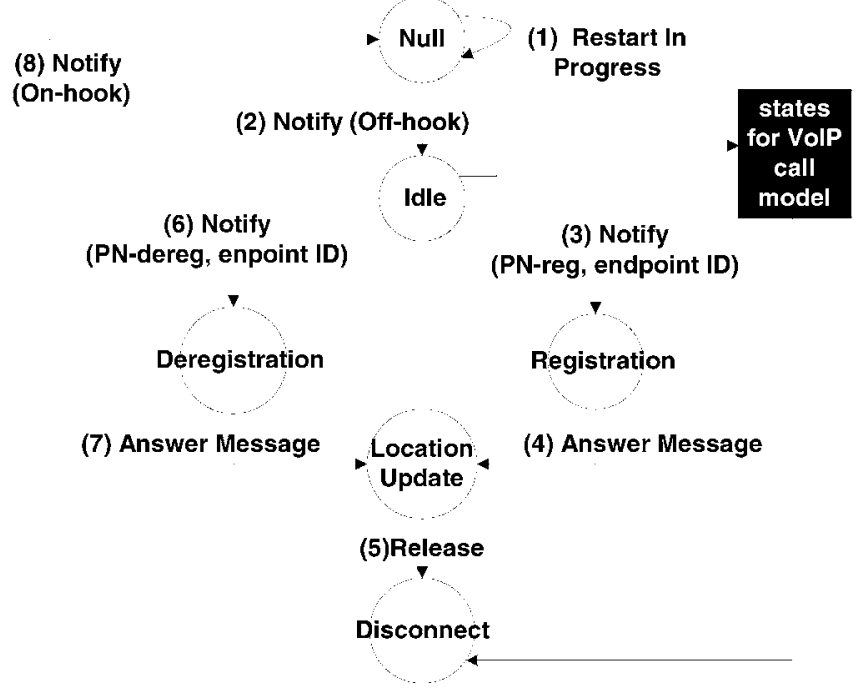

Fig. 16. The AFS finite state machine.

telephone. The endpoint ID in the message is the telephone number of that fixed telephone.

Step 3. Upon receipt of the Registration message, the RGW invokes a Notify command with event type Off-hook to the CA. The call state of the CA changes from Null to Idle (Transition 2 in Fig. 16). The CA replies with a Notification Request message, which contains a digit map to instruct the RGW to provide the dialed number.

Step 4. The RGW sends a Notify command to the CA. The Notify command contains a PN-reg parameter and the endpoint ID. The PN-reg parameter is a command code (which represents AFS Registration) concatenated with the PN. The CA changes state from Idle to Registration (Transition 3 in Fig. 16).

Step 5. The CA updates the PN record in the Follow-me database by sending an SS7 Initial Address Message (IAM) to PSTN through the signaling gateway (signaling path (a) $\rightarrow$ (b) $\rightarrow$ (c) in Fig. 6). The IAM message is used to reserve a call path from the AFS to the PSTN. This message contains the called party number and the calling party number. The calling party number is the endpoint ID. The called party number points to the Follow-me application in the PSTN. For example, if the Follow-me service is provided by the Chunghwa Telecom (CHT), the called party number is 080993993. This number points to the Follow-me application offered by the CHT.

Step 6. When the call is connected to the Follow-me application, the PSTN responds with the SS7 Address Complete Message and then the SS7 Answer Message to the CA (path (c) $\rightarrow$ (b) $\rightarrow$ (a) in Fig. 6). These two SS7 messages indicate that the communication path between the TGW in the VoIP network and the Follow-me application has been established (trunk path (d) $\leftrightarrow$ (f) in Fig. 6). The CA changes state from Registration to Location Update (Transition 4 in Fig. 16).

Step 7. The CA instructs the TGW to send the PN and the PIN (Personal Identification Number) to the Follow-me application. The PIN is used for authentication to 
$\begin{array}{llll}\text { AP1 RGW } & \text { CA } & \text { PSTN }\end{array}$

\begin{tabular}{l} 
AFS initialization \\
(1) Restart In Progress \\
\hline (2) Registration (PN, endpoint ID) \\
(1) Notification Request (Off-hook) \\
(3) Notify (Off-hook) \\
(3) Notification Request (digit map) \\
(4) Notify (PN-reg, endpoint ID)
\end{tabular}

AFS Deregistration procedure

(10) Deregistration (PN, endpoint ID)

-

(11) Notify (Off-hook)

(11) Notification Request (digit map)

(12) Notify (PN-dereg, endpoint ID)

(16) Deregistration Accept (PN, endpoint ID)

(17) Deregistration Complete
(16) Notification Request (On-hook)

\section{(17) Notify (On-hook)}

(17) Notification Request ( Off-hook)
(13) Initial Address Message

(14) Address Complete Message

(14) Answer Message

(15) Dial (PN,PIN)

(15) Release

(15) Release Complete

Fig. 17. AFS registration and deregistration message flows.

prevent illegal users from accessing the Follow-me application. After authentication is completed, the TGW sends some control digits to the Follow-me application, which activates the PN record modification. In CHT Follow-me service, the control digits are 111 . The Followme application then stores the calling party number received from the IAM message in Step 5 into the PN record. At this point, the Follow-me database update is complete and the AFS application disconnects the call path by sending an SS7 Release message to the CA. The CA replies an SS7 Release Complete message and moves to the Disconnect state (Transition 5 in Fig. 16).
Step 8. To inform AP1 of registration completion, the CA sends the Notification Request command to the RGW, which requests the RGW to detect the registration complete response from AP1 (this response is an Onhook event). The RGW sends the Registration Accept message to AP1. AP1 considers the registration is successful and replies with the Registration Complete message.

Step 9. The RGW invokes a Notify command with event type On-hook to the CA, which acknowledges the completion of the Registration procedure. Upon receipt of this Notify command, the CA changes state from Disconnect to 


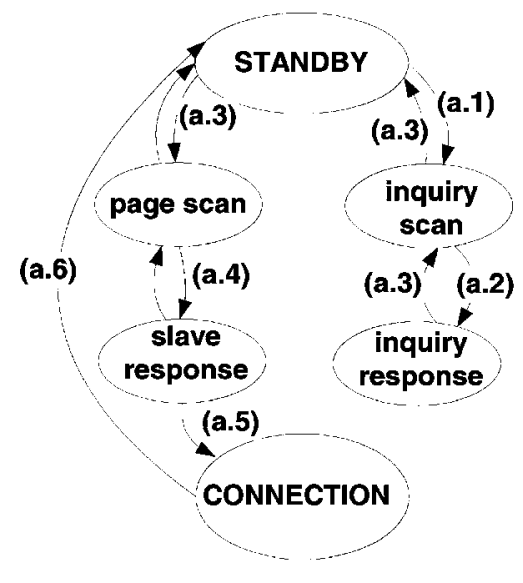

(a)

Fig. 18. AFS link controller finite state machine.

Null (Transition 8 in Fig. 16). As in Step 1, the CA sends a Notification Request command to the RGW. This command instructs the RGW to detect the next Off-hook event and the AFS repeats the whole process for the next registration/deregistration.

Steps 10-17 describe the AFS Deregistration procedure. These steps are similar to the AFS Registration procedure except for the following: In Step 12, the command code of the message is PN-dereg (which stands for deregistration). Furthermore, upon receipt of the Notify command, the CA changes state from Idle to Deregistration (Transition 6 in Fig. 16). In Step 13, the calling party address of the IAM message is set to a default number (e.g., the cellular phone number or the secretary phone number of the user).

\section{APPENDIX B}

\section{The BLuetooth Registration AND Deregistration Procedures}

In our AFS implementation, the Bluetooth Link Controller functions are utilized for paging, inquiry, and polling. AFS also uses the Link Manager function for mode selection (to reduce power consumption; we will elaborate on this aspect later). The Link Controller maintains a finite state machine for connection establishment. Based on the state of the machine, a particular link control function is executed. In this machine, two major states are defined for both master and slave: STANDBY and CONNECTION. In the STANDBY state, the Bluetooth device operates in a low power mode, which has no connection with other Bluetooth devices. In the CONNECTION state, connection is established and the device may or may not transmit packets. Besides these two major states, seven substates are defined to manage connection between two Bluetooth devices. For example, to initiate a new connection, the master changes state from STANDBY to CONNECTION through the inquiry, page, and master response substates. On the other hand, the slave changes from STANDBY to CONNECTION through the inquiry scan, inquiry response, page scan, and slave response substates. The state

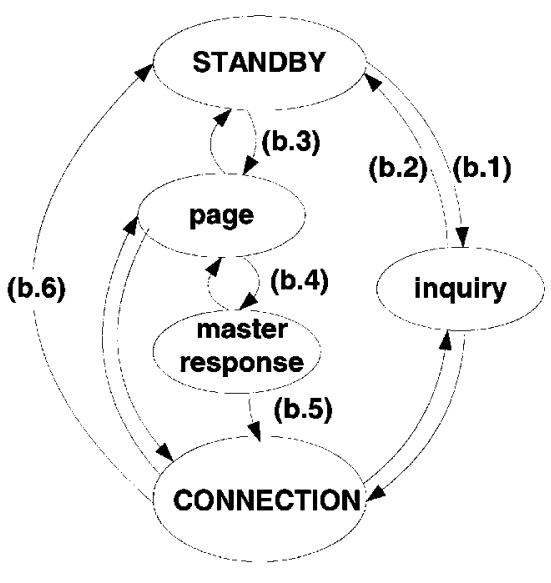

(b) diagrams of the Link Controller finite state machine are illustrated in Fig. 18.

Based on the above link controller finite state machine, we describe the AFS radio tracking procedures. When a PD moves into a piconet, the Link Controller state machine of the AP is either in the STANDBY state (no PD is in the piconet) or the CONNECTION state (at least one PD is in the piconet). For description purposes, we consider the situation that no PD is in the piconet and the first PD is moving into this piconet. At the beginning of this scenario, both the AP and the PD are in the STANDBY state. The polling mechanism is exercised between the AP and the PD, as described in the following steps (see Fig. 19).

Step 1: PD Detection I. Through HCI, the AP AFS application instructs the AP Bluetooth module to periodically check if any new PDs have moved into the piconet. In this detection phase, the AP changes state from STANDBY to inquiry (Transition b.1 in Fig. 18), which triggers an inquiry message broadcast (Message 1 in Fig. 19) in the piconet.

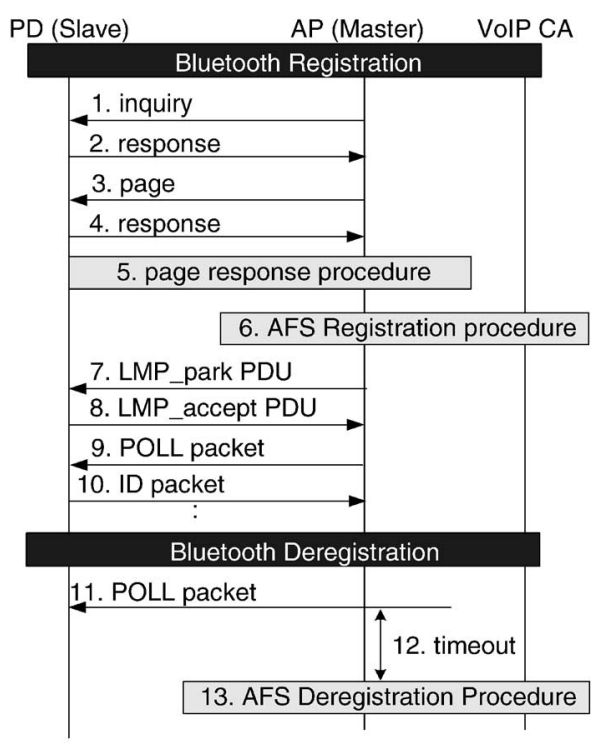

Fig. 19. Bluetooth registration and deregistration message flows. 
On the other hand, the PD periodically listens to the inquiry message. In this listening phase, the PD changes state from STANDBY to inquiry scan (Transition a.1 in Fig. 18).

Step 2: PD Detection II. When the PD receives an inquiry message in the inquiry scan substate, it changes to the inquiry response substate (Transition a.2 in Fig. 18) and returns its ID to the AP (Message 2 in Fig. 19). The PD changes to the page scan substate (Transitions a.3 in Fig. 18) and expects to receive a page message from the AP. Upon receipt of the inquiry response message, the AP changes to the STANDBY state (Transition b.2 in Fig. 18). Then, the AP proceeds to establish signaling path to the PD in the next step.

Step 3: Signaling Path Establishment I. The AP changes state from STANDBY to page (Transition b.3 in Fig. 18) and transmits the page message to the PD (Message 3 in Fig. 19).

Step 4: Signaling Path Establishment II. After Step 2, the PD is in the page scan substate. Upon receipt of the page message (which is issued by the AP in Step 3), the PD changes state from page scan to slave response (Transition a.4 in Fig. 18) and initiates the page response procedure by sending its ID to the AP (Message 4 in Fig. 19).

Step 5: Signaling Path Establishment III. When the AP receives the response message, it changes to the master response state (Transition b.4 in Fig. 18), and exchanges four messages with the PD to establish the connection for data transmission (details of this procedure can be found in [3]). At the end of this step, the signaling path is established, and both the AP and the PD switch to the CONNECTION state (Transitions a.5 and b.5 in Fig. 18).

Step 6: Registration. The AP invokes AFS registration procedure (see Section 2) to update the PD's PN record in the Follow-me database.

Step 7: Power Saving I. In Bluetooth, a device can be in one of four operation modes [3], [19] and two of them (i.e., active and park) are utilized in the AFS system. The active mode is the default mode used in the beginning of the AP-PD connection. In this mode, at most seven active PDs can be accommodated in a piconet. On the other hand, the park mode is the low-power mode, which allows the AP to connect to more than seven PDs. A parked PD wakes up at regular intervals to listen to the POLL message broadcast from the AP. Note that, in the active mode, the PD consumes $40-60 \mathrm{~mA}$, while, in the park mode, it consumes $0.55 \mathrm{~mA}$. At the end of Step 5, the PD operates in the active mode. To reduce power consumption of the PD, the AP instructs the PD to switch to the park mode by sending an LMP_park PDU (Message 7 in Fig. 19).

Step 8: Power Saving II. Upon receipt of the LMP_park PDU, the PD switches to the park mode. The PD sends an LMP_accepted PDU to the AP (Message 8 in Fig. 19) to indicate that it has operated in the park mode.

Steps 9 and 10: Subsequent Polling. After Step 8, both the AP and the PD are in the CONNECTION state. The AP periodically polls the PD to check if the PD is still in the piconet (Message 9 in Fig. 19). The PD acknowledges the polling by returning an ID packet [3] (Message 10 in Fig. 19).
Step 11: Deregistration. After sending a POLL packet, if the AP does not receive the response packet from the PD within a timeout period (see message 11 and period 12 in Fig. 19), it implies that the PD has left the AFS service area. In this case, the AP invokes the deregistration procedure (see Section 2). If all PDs have left the piconet, the AP changes state from CONNECTION to STANDBY (Transition b.6 in Fig. 18). Similarly, if the PD does not receive the POLL packet, the PD knows that it is no longer in the piconet. The PD switches from the CONNECTION state to the STANDBY state (Transition a.6 in Fig. 18). At this point, both the AP and the PD exercise Step 1 of the polling procedure again.

\section{ACKNOWLEDGMENTS}

The authors would like to thank the three anonymous reviewers. Their comments have significantly improved the quality of this paper. This work was sponsored in part by MOE Program of Excellence Research under contract 90-EFA04-4, TAHOE Network, FarEastone, National Science Council under contract NSC 90-2213-E-009-156, the Lee and MTI Center for Networking Research, NCTU.

\section{REFERENCES}

[1] I.F. Akyildiz, J. McNair, J.S.M. Ho, H. Uzunalioglu, and W. Wang, "Mobility Management in Next Generation Wireless Systems," IEEE Proceedings, vol. 87, no. 8, pp. 1347-1385, 1999.

[2] M. Arango, A. Dugan, I. Elliott, C. Huitema, and S. Pickett, "Media Gateway Control Protocol," Technical Report RFC 2705, Internet Eng. Task Force, 1999.

[3] Bluetooth Special Group, "Specification of the Bluetooth System, V1.0B," 1999.

[4] V.-A. Bolotin, "Modeling Call Holding Time Distributions for CCS Network Design and Performance Anallysis," IEEE J. Selected Areas in Comm., vol. 14, no. 4, 1996.

[5] ETSI, "Radio Equipment and Systems (RES); Digital Enhanced Cordless Telecommunications (DECT); Common Interface (CI)," Technical Report ETS 300 175, ETSI, 1996.

[6] Y. Fang and I. Chlamtac, "Teletraffic Analysis and Mobility Modeling for PCS Networks," IEEE Trans. Comm., vol. 47, no. 7, pp. 1062-1072, July 1999.

[7] Y. Fang, I. Chlamtac, and Y.-B. Lin, "Modeling PCS Networks under General Call Holding Time and Cell Residence Time Distributions," IEEE/ACM Trans. Networking, vol. 5, no. 6, pp. 893906, 1998.

[8] R. Guerin, "Channel Occupancy Time Distribution in a Cellular Radio System," IEEE Trans. Vehicular Technology, vol. 36, pp. 89-99, Aug. 1987.

[9] A. Harter and A. Hopper, "A Distributed Location System for the Active Office," IEEE Network Magazine, pp. 62-70, Jan. 1994.

[10] ITU-T, "Operations and Quality of Service Universal Personal Telecommunication (UPT),"technical report, ITU-T, 1993.

[11] F.P. Kelly, Reversibility and Stochastic Networks. John Wiley \& Sons, 1979.

[12] Y.-B. Lin, "Eliminating Overflow for Large-Scale Mobility Databases in Cellular Telephone Networks," IEEE Trans. Computers, vol. 50, no. 4, pp. 356-370, Apr. 2001.

[13] Y.-B. Lin and Y.-K. Chen, "Reducing Authentication Signaling Traffic in Third Generation Mobile Network," IEEE Trans. Wireless Comm., 2002. See also http://liny.csie.nctu.edu.tw/\\#jorunal.

[14] Y.-B. Lin and I. Chlamtac, Wireless and Mobile Network Architectures. John Wiley \& Sons, 2001.

[15] R. Mettale, "Bluetooth Protocol Architecture," technical report Version 1.0, Nokia, 1999.

[16] L. Ong et al., "Framework Architecture for Signaling Transport," Technical Report RFC 2719, Internet Eng. Task Force, 1999.

[17] A.-C. Pang, P. Lin, and Y.-B. Lin, "Modeling Mis-Routing Calls Due to User Mobility in Wireless VoIP," IEEE Comm. Letters, vol. 4, no. 12, pp. 394-397, 2001. 
[18] R.F. Rey, Engineering And Operations in the Bell System. AT\&T Bell Laboratories, 1989.

[19] G.V. Záruba, S. Basagni, and I. Chlamtac, "Bluetrees-Scatternet Formation to Enable Bluetooth-Based Ad Hoc Networks," Proc. IEEE Int'l Conf. Computings (ICC 2001), 2001.

[20] M. Zorzi, "Energy Management in Personal Communications and Mobile Computing," IEEE Personal Comm., vol. 5, 1998.

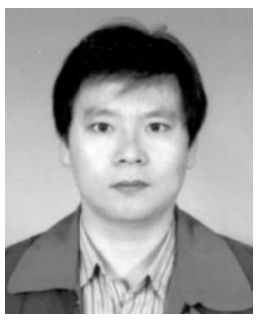

Yi-Bing Lin received the BSEE degree from National Cheng Kung University in 1983 and the $\mathrm{PhD}$ degree in computer science from the University of Washington in 1990. From 1990 to 1995, he was with the Applied Research Area at Bell Communications Research (Bellcore), Morristown, New Jersey. In 1995, he was appointed a professor in the Department of Computer Science and Information Engineering (CSIE), National Chiao Tung University (NCTU). In 1996, he was appointed deputy director of the Microelectronics and Information Systems Research Center, NCTU. During 1997-1999, he was elected chairman of CSIE, NCTU. His current research interests include design and analysis of personal communications services network, mobile computing, distributed simulation, and performance modeling. Dr. Lin is an associate editor of IEEE Network, an editor of the IEEE Journal of Systems and Circuits: Wireless Series, an editor of IEEE Personal Communications Magazine, an editor of Computer Networks, an area editor of the ACM Mobile Computing and Communication Review, a columnist of the ACM Simulation Digest, an editor of the International Journal of Communications Systems, an editor of $A C M /$ Baltzer Wireless Networks, an editor of Computer Simulation Modeling and Analysis, an editor of the Journal of Information Science and Engineering, program chair for the Eighth Workshop on Distributed and Parallel Simulation, general chair for the Ninth Workshop on Distributed and Parallel Simulation. program chair for the Second International Mobile Computing Conference, guest editor for the ACM/Baltzer MONET special issue on personal communications, a guest editor for the IEEE Transactions on Computers special issue on mobile computing, and a guest editor for IEEE Communications Magazine special issue on active, programmable, and mobile code networking. He is the author of the book Wireless and Mobile Network Architecture (coauthor with Imrich Chlamtac, John Wiley \& Sons). He received the 1998 and 2000 Outstanding Research Awards from the National Science Council, Republic of China and 1998 Outstanding Youth Electrical Engineer Award from CIEE, ROC. He is an adjunct research fellow of the Academia Sinica. He is a senior member of the IEEE.

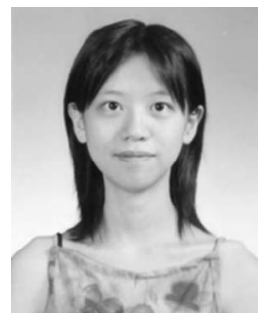

Hsu-Yung Cheng received the BS degree in computer science and information engineering from National Chiao Tung University in 2000 and the MS degree from the same department in 2002. Her current research interests include personal communications services network, wireless telechology, and Voice over IP technology.

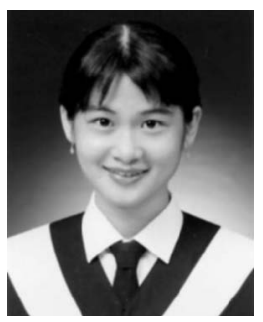

Ya-Hsing Cheng received the BSCSIE and MSCSIE degrees from National Chiao Tung University in 2000 and 2002, respectively. Her current research interests include personal communications services and wireless communications.

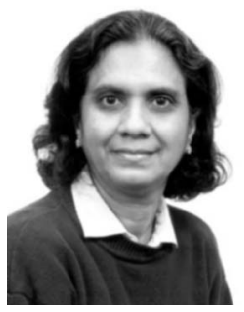

Prathima Agrawal received the $\mathrm{PhD}$ degree in electrical engineering from the University of Southern California. She is assistant vice president of the Internet Architecture Research Laboratory and executive director of the Computer Networking Research Department at Telcordia Technologies, Morristown, New Jersey. She is an adjunct professor of electrical and computer engineering at Rutgers University, New Jersey. She worked for 20 years at AT\&T/ Lucent Bell Laboratories in Murray Hill, New Jersey, as head of the Networked Computing Research Department. She leads the ITSUMO research project focusing on wireless IP networking. Her research interests are computer networks, mobile and wireless computing, and communication systems. She has published more than 150 papers and has received or applied for more than 50 US patents. She is the recipient of the Distinguished Member of Technical Staff Award of AT\&T Bell Laboratories, 1985, the Telcordia CEO Award, 2000, and the 2001 SAIC ESTC (Executive Science and Technology Council) Publication Award. Dr. Agrawal is a fellow of the IEEE and a member of the ACM. She is the recipient of the IEEE Computer Society's Distinguished Service Award, 1990, and the IEEE Third Millennium Medal, 2000. She chaired the IEEE Fellow Selection Committee during 1998-2000.

$\triangleright$ For more information on this or any computing topic, please visit our Digital Library at http://computer.org/publications/dlib. 